\title{
Old-Boy Network. Militärdienst und ziviler Berufserfolg in der Schweiz
}

\author{
Old-Boy Network. Military Service and Professional Success in Civilian Life in Switzerland
}

\author{
Ben Jann* \\ Institut für Soziologie, Universität Bern, Lerchenweg 36, CH-3000 Bern 9
}

\begin{abstract}
Zusammenfassung: Eine zumindest bis vor kurzem in der Schweiz noch populäre Ansicht lautet, dass eine Karriere in der Armee den beruflichen Erfolg im zivilen Leben stark befördert. Obwohl militärische und berufliche Karriere als Besonderheit des schweizerischen Milizsystems im biografischen Verlauf miteinander verschränkt sind, bleibt aber der tatsächliche Einfluss der militärischen Karriere auf den Berufserfolg empirisch noch nachzuweisen. Zudem ist auch die Frage nach den möglichen Gründen zu stellen. Ziel dieses Beitrags ist also erstens die empirische Prüfung des positiven Effekts der militärischen auf die zivile Karriere. Zweitens sollen mögliche Ursachen gegenübergestellt werden. Als solche werden insbesondere Selbstselektion, der Erwerb von Humankapital (Führungskompetenzen) und der Aufbau eines wirkungsvollen Beziehungsnetzes in Betracht gezogen. Die Analysen anhand der Daten des Schweizer Arbeitsmarktsurveys 1998 zeigen erstens, dass höhere militärische Ränge auch bei Kontrolle von Bildung und Berufserfahrung mit erhöhtem Erwerbseinkommen einhergehen. Zweitens zeigt sich, dass dieser Einkommensbonus allem Anschein nach weder auf Mechanismen der Selbstselektion noch auf dem Erwerb von besonders gewinnbringendem Humankapital beruht. Vielmehr ist zu vermuten, dass der größere Berufserfolg von Milizoffizieren mit ihrem an „wertvollen“ Beziehungen reichen sozialen Netzwerk zusammenhängt.
\end{abstract}

\section{Einleitung}

Das militärische Milizsystem in der Schweiz sieht für Männer über eine längere Dauer des Erwachsenenlebens eine allgemeine Wehrpflicht vor. Nach einer mehrmonatigen Grundausbildung (Rekrutenschule) im 20. Lebensjahr werden die Wehrpflichtigen je nach Dienstgrad bis zu einem Alter von 42 bis 52 Jahren i.d.R. zweijährlich zu einem dreiwöchigen Wiederholungskurs einberufen. Hinzu kommen weitere Kurse und Einsätze für höhere Dienstgrade. Da Berufsoffiziere äußerst selten sind, der Großteil des militärischen Kaders also einem zivilen Beruf nachgeht, stellt sich die Frage nach der Verflechtung von militärischer und ziviler Karriere.

Auch heute noch wird in weiten Teilen der schweizerischen Bevölkerung die Meinung vertreten, dass

\footnotetext{
* Gefördert durch den Schweizerischen Nationalfonds (SNF) im Rahmen des Schwerpunktprogramms „Zukunft Schweiz“ (Projekt Nr. 5004-47887). Für Anregungen und kritische Hinweise danke ich Jürg Arpagaus, Andreas Diekmann, Axel Franzen, Thomas Gautschi, Christoph Kopp, Peter Preisendörfer, Werner Raub und Stefan Wehrli, den Teilnehmern und Teilnehmerinnen der Tagung „Soziale Netzwerke“ der Sektion „Modellbildung und Simulation" der Deutschen Gesellschaft für Soziologie in München (21.-23. März 2002) sowie zwei anonymen Gutachtern bzw. Gutachterinnen.
}

sich eine militärische Karriere vorteilhaft auf die zivile berufliche Laufbahn auswirkt (Haltiner et al. 2001), was sich in Sprichwörtern wie „Gold am Hut tut der Karriere gut!“ (Hochstrasser 1989: 1) niedergeschlagen hat. Auch liefern Befragungen von Führungskräften in der Schweizer Wirtschaft deutliche Hinweise. So schreibt etwa Spälti (1995: 23), „... dass überdurchschnittlich viele Führungskräfte von Schweizer Unternehmen auch in der Armee Kaderfunktionen einnehmen. Ein tradiertes Klischee besagt sogar, dass in Schweizer Unternehmen nur Karriere machen könne, wer auch im Militär Offizier geworden sei. “ Oder Staffelbach (1994: 9): „Mehr als die Hälfte der (männlichen) Schweizer Führungskräfte sind Offiziere.“ Und: „Drei Viertel der (Miliz-)Generalstabsoffiziere sind im Topmanagement" (ähnliche Angaben bei Hollenstein 1987, Hochstrasser 1989). Dass die militärische Position ein Kriterium bei der Stellenbesetzung sein kann, zeigen zudem Befragungen von Personalchefs. So etwa die Befunde von Berner (1994: 79): „Bei der Besetzung von oberen und mittleren Führungspositionen wird in fast zwei Dritteln der Fälle nach dem militärischen Grad des Stellenbewerbers gefragt" (vgl. auch Bertossa et al. 1994, Zimmermann et al. 1992). Weiterhin ist bekannt, dass Personen in höheren Positionen der öffentlichen Verwaltung und in der politischen Elite häufig auch eine militärische Karriere vorweisen können. So 
Ambühl (1998): „In Basel ... verfügen auffällig viele höhere Staatsdiener gleichzeitig auch über ein Offizierspatent“, oder Kriesi (1980: 533): „Eine militärische Karriere ist also für Akteure im inneren Kreis der politischen Elite, wenn nicht Voraussetzung, so doch üblich.“

In neuerer Zeit regen sich allerdings bezüglich der Vorteilhaftigkeit einer höheren militärischen Position durchaus auch kritische Stimmen („Die Wirtschaft pfeift immer lauter auf militärische Führungsqualifikationen“, Lutz 1998; vgl. auch Vontobel 1992, Lichtsteiner 1997, Erb 1997, Staffelbach 1998, Schmitt 1992, Keller 1995). Insbesondere die häufigen, durch militärische Verpflichtungen bedingten Absenzen von Milizoffizieren seien für Unternehmen eine Belastung (Oester 1996: 55). So schreibt etwa die Neue Zürcher Zeitung (27. Januar 1999: 67), dass „... bei höheren Offizieren vor allem die langen Abwesenheiten ab dem 35. Altersjahr negativ ins Gewicht [fallen]. Für solche Absenzen zeigen namentlich multinationale Firmen wenig Verständnis.“ $\mathrm{Zu}$ einem ähnlichen Schluss kommen Bertossa et al. (1994: 65) in einer Befragung von Personalchefs: „Negativ ins Gewicht fallen - verstärkt als früher - die mit der Weiterbildung verbundenen absenzbedingten Kosten." So hat auch der Prestigewert einer milizmilitärischen Karriere gemäß Bevölkerungsumfragen in den 1990er Jahren deutlich abgenommen. Haltiner et al. (2001: 147) schreiben: „Eines der bis heute nachgewiesenermaßen wichtigsten Karrieremotive - der zivile Nutzen des Weitermachens ${ }^{1}$ - steht im Begriffe, weiter an Zugkraft zu verlieren. Der Rückgang von $85 \%$ Zustimmung im Jahre 1983 auf $60 \%$ in diesem Jahr [2001] spricht eine deutliche Sprache." ${ }^{2} \mathrm{Ob}$ wohl dieser Rückgang wohl zumindest teilweise die tatsächliche Veränderung des Einflusses einer militärischen Karriere auf das zivile Berufsleben widerspiegelt, muss gleichwohl auch weiterhin mit diesem Einfluss - evtl. in abgeschwächter Form gerechnet werden. Hinzu kommt, dass sich im

\footnotetext{
1 „Weitermachen“ ist ein in der Schweiz gebräuchlicher Begriff und meint: sich zum Unteroffizier oder Offizier ausbilden lassen.

${ }^{2}$ Gemeint ist der Anteil Personen, die sich mit der Aussage „Eine militärische Führungserfahrung bringt auch im Zivilen berufliche Vorteile" sehr oder eher einverstanden erklärten. Neben den angesprochenen Werten wurden für die Jahre 1995 und 1998 Anteile von $73 \%$ beziehungsweise $66 \%$ gemessen. Gleichzeitig wurde für die Jahre 1998 und 2001 allerdings auch ein leichter Rückgang der Zustimmung zur Aussage registriert, dass sich eine militärische Karriere nachteilig auf die Arbeitsmarktchancen auswirke (Haltiner et al. 2001: 147).
}

Querschnitt ein großer Anteil der erwerbstätigen Personen in einem mehr oder weniger fortgeschrittenen Stadium der beruflichen Karriere befindet und sich die befördernde Wirkung des militärischen Rangs schon einige Zeit zuvor eingestellt haben dürfte. Ein positiver Effekt der militärischen Karriere auf den beruflichen Erfolg sollte also - so es ihn denn je gegeben hat - in einer Querschnittsbetrachtung auch heute noch nachgewiesen werden können. $\mathrm{Zu}$ bemerken ist allerdings, dass aufgrund des retrospektiven Charakters der Analyse von einem solchen Ergebnis nur eingeschränkt auf die künftige Wirkungsweise bei den eher am Anfang ihrer Berufskarriere stehenden Generationen geschlossen werden kann.

Über anekdotische Hinweise und Fallstudien hinaus ist mir keine Untersuchung mit landesweit repräsentativen Daten bekannt, die den erwähnten $\mathrm{Zu}$ sammenhang systematisch erforscht hätte. In diesem Beitrag soll nun der gemäß den eingangs zitierten Befunden zu erwartende positive Einfluss der militärischen Karriere auf den beruflichen Erfolg erstmals für die gesamte Schweiz statistisch geprüft werden.

Sollte sich auch unter Kontrolle der üblichen Bestimmungsgrößen tatsächlich ein positiver Effekt zeigen, so bieten sich drei Erklärungsalternativen an. Erstens kann es sich um ein Phänomen der Selbstselektion handeln. Das heißt, Personen sind aus den gleichen Gründen beruflich erfolgreich, die sie auch beim Militär erfolgreich sein lassen, nämlich weil sie (1) eine erfolgreiche Karriere im Allgemeinen (also sowohl in Militär als auch Beruf) als wichtiger erachten als andere oder (2) von vornherein mit für beide Bereiche günstigeren Eigenschaften ausgestattet sind. Zum Beispiel weisen Mueller und Mazur (1996) darauf hin, dass das physische Erscheinungsbild einer Person - in ihrer Studie ein dominanter versus unterwürfiger Gesichtsausdruck - einen Einfluss auf die militärische Karriere haben kann. Ähnlich ist bekannt, dass auch bei der Rekrutierung von Führungskräften physische Eigenschaften wie etwa die Körpergröße eine Rolle spielen (Hartmann 1996: 118ff.). Die Möglichkeit von Selektionseffekten durch das äußere Erscheinungsbild oder auch durch weitere Eigenschaften wie Charaktermerkmale u. ä. muss hier allerdings mangels geeigneter Daten außer Betracht gelassen werden. Es verbleibt die unter (1) aufgeführte Erklärung mit Hilfe der Karrieremotivation. Gemäß der Selbstselektionshypothese kommt der Effekt der militärischen Position also dadurch zustande, dass Personen, die eine militärische Karriere einschlagen, auch mehr in den zivilen Berufs- 
erfolg investieren. Als Brückenhypothese nehme ich an, dass das Ausmaß an Arbeitseinsatz oder -anstrengung etwas über die subjektive Wichtigkeit einer erfolgreichen Karriere aussagt.

Zweitens könnte der Karriereeffekt auf spezielle, im Rahmen der militärischen Weiterbildung und Praxis erworbene Fähigkeiten zurückzuführen sein, mithin auf zusätzliche Investitionen ins Humankapital (Becker 1993), die zu komparativen Vorteilen im Beruf verhelfen. Besonders von militärischer Seite wird oft betont, dass die militärische Führungsausbildung und -erfahrung auch im zivilen Erwerbsleben nützlich sei und von der Wirtschaft nachgefragt werde: „Synergien zwischen ziviler und militärischer Führung sind offensichtlich " (Staffelbach 1994: 9; vgl. auch Spälti 1990, 1995, Keller/ Wigger 1998, Brunner 1997, Fopp 1992). Ähnliches fördert eine Befragung von Personalchefs zu Tage: „Positiv gewertet werden die potentiellen Führungserfahrungen ..." (Bertossa et al. 1994: 65). Die Humankapitalhypothese besagt also, dass die Milizoffiziere in der militärischen Ausbildung und Praxis besondere Führungskompetenzen erwerben, die im Wirtschaftsleben gefragt sind und somit zu verbesserten Arbeitsmarktchancen führen. Die These ist allerdings nur bedingt plausibel, da, wie bereits erwähnt, vor allem in neuerer Zeit die häufigen mit dem Militärdienst einhergehenden Absenzen vom Arbeitsplatz den Nutzen der militärischen Führungskompetenzen schmälern dürften; überdies wird bisweilen auch die Vereinbarkeit militärischer und ziviler Führungsgrundsätze angezweifelt. Ferner muss man bedenken, dass Personen, die keine militärische Führungsausbildung absolvieren, ähnliche Kompetenzen anderweitig erwerben können.

Ein dritter Erklärungsansatz schließlich stützt sich auf das Konzept des Sozialkapitals (Bourdieu 1983, Coleman 1988). Was in den Vereinigten Staaten das Old-Boy Network von Hochschulabsolventen ist so die These -, sind in der Schweiz die sozialen Beziehungen, die sich während des Militärdienstes entwickeln. Besonders mit dem Aufstieg in das militärische Kader ergeben sich viele Möglichkeiten zu Bekanntschaften, die sich - obwohl eigentlich nicht als enge Freundschaften zu charakterisieren - durch ein hohes Maß an Loyalität und Vertrauen auszeichnen (Oester 1996: 58ff.; vgl. auch Ambühl 1998) und die während der Dienstpflicht bis in ein relativ hohes Alter regelmäßig wieder aufgefrischt werden. Die so entstehenden Netzwerke finden in den Offiziersgesellschaften sogar ihre institutionalisierte Verwirklichung. Die Annahme liegt nahe, dass über diese Beziehungen u. a. auch wertvolle Informationen bezüglich des Wirtschaftslebens (z.B. über offene Stellen) transportiert werden (Granovetter 1973). Gemäß der Sozialkapitalbypothese ist der begünstigende Effekt der militärischen Karriere für den zivilen Beruf also mit dem Zugang zu einem besonderen sozialen Netzwerk zu erklären, das im Kontext der Stellensuche und -besetzung Erfolg versprechend aktiviert werden kann.

Ich werde im nächsten Abschnitt (2. ) kurz auf die Datengrundlage meiner Untersuchungen sowie auf einige methodische Aspekte eingehen, worauf in Abschnitt 3 die empirische Überprüfung der Hypothesen folgt. Den Abschluss (4.) bildet eine kritische Diskussion und Zusammenfassung der zentralen Ergebnisse.

\section{Daten und Methode}

Die Grundlage für die folgenden Analysen bilden die Daten des Schweizer Arbeitsmarktsurveys 1998 (SAMS 98) - einer durch den Schweizerischen Nationalfonds geförderten Bevölkerungsumfrage im Rahmen eines Gemeinschaftsprojekts der Institute für Soziologie und Politikwissenschaft der Universität Bern. Dem Survey liegt eine Zufallsstichprobe der Schweizer Wohnbevölkerung des Jahres 1998 im Alter von 18 bis 70 Jahren zugrunde $(\mathrm{N}=3028)$. Die Daten wurden mit Hilfe eines standardisierten Telefoninterviews und einer schriftlichen Nachbefragung erhoben (zu den Details der Befragung siehe Diekmann et al. 1999 und Jann 2003). ${ }^{3}$

Der Survey enthält eine Vielzahl von Informationen vor allem in Zusammenhang mit dem Erwerbsleben. Unter anderem wurden aber auch Fragen bezüglich der militärischen Karriere gestellt. Von der betroffenen Teilpopulation (Männer mit Schweizer Staatsbürgerschaft) haben insgesamt $79 \%$ angegeben, Militärdienst zu leisten bzw. geleistet zu haben (Tabelle 1). Entsprechend der hierarchischen Organisationsstruktur des Militärs treten höhere militärische Ränge relativ selten auf. So machen in der Stichprobe der Wehrpflichtigen und Entlassenen (also der Personen, die Militärdienst leisten bzw. geleistet haben) die Offiziere ${ }^{4}$ nur knapp $8 \%$ aus. Der Löwenanteil entfällt auf Soldaten und Unter-

\footnotetext{
${ }^{3}$ Der SAMS 98 kann für Sekundäranalysen bei SIDOS in Neuchâtel bezogen werden (www.sidos.ch, Referenznummer 5306). Ein Codebuch und weitere Informationen findet man zudem unter http://www.soz.unibe.ch/forschung/ wl/daten.asp.

${ }^{4} \mathrm{Zu}$ den Offizieren zählen in absteigender Rangfolge: höhere Stabsoffiziere (Korpskommandant, Divisionär, Bri-
} 
Tabelle 1 Militärdienst und Verteilung militärischer Ränge

\begin{tabular}{|c|c|c|c|c|}
\hline & Prozent & & Prozent & Sollbestand $2001^{a}$ \\
\hline \multirow[t]{6}{*}{ leistet(e) Militärdienst } & 79.3 & als: & & \\
\hline & & - Soldat/Hilfsdienst & 74.5 & 75.5 \\
\hline & & - Unteroffizier & 17.8 & 15.7 \\
\hline & & - Leutnant, Oberleutnant & 4.3 & 88 \\
\hline & & - Hauptmann oder höher & 3.4 & 8.8 \\
\hline & & Total & 100.0 & 100.0 \\
\hline \multirow[t]{5}{*}{ leistet(e) keinen Militärdienst } & 20.7 & weil: & & \\
\hline & & - untauglich & 78.1 & \\
\hline & & - verweigert & 5.4 & \\
\hline & & - noch nicht ausgehoben & 16.5 & \\
\hline & & Total & 100.0 & \\
\hline Total & 100.0 & & & \\
\hline
\end{tabular}

Anmerkungen: Männer mit Schweizer Staatsbürgerschaft im Alter von 18-70 Jahren; N=1201; Quelle: SAMS 98, ungewichtet.

a Prozentuale Anteile am Sollbestand der Schweizer Armee (Stand 2001) gemäss VBS (Eidgenössisches Departement für Verteidigung,

Bevölkerungsschutz und Sport; Quelle: http://www.vbs-ddps.ch/internet/vbs/de/home/publikationen/zahlen.html, 13.08.2002).

offiziere. Dies liegt etwa im Rahmen des Sollbestandes der Armee, der für das Jahr 2001 einen Offiziersanteil von knapp $9 \%$ ausweist (vgl. ebenfalls Tabelle 1, wobei sich die $9 \%$ natürlich nur auf aktuell Wehrpflichtige beziehen). Bei den Befragten, die keinen Militärdienst leisten bzw. geleistet haben, handelt es sich - abgesehen von Frauen und Ausländern - in der Hauptsache um Ausgemusterte („Untaugliche“).

Berufserfolg hat viele Facetten. Um der Frage des Einflusses des militärischen Rangs auf den Berufserfolg nachzugehen, wäre z.B. die Analyse der erreichten beruflichen Position nahe liegend. Leider wurde diese in den verwendeten Daten nur grob erfasst, was die Aussagekraft einer solchen Analyse stark einschränkt. In Betracht gezogen wurde weiterhin eine Operationalisierung des Berufserfolgs über das mit dem Beruf verbundene soziale Prestige (nach Ganzeboom/Treiman 1996). Es zeigt sich jedoch auch hier, dass gerade in den höheren Positionen erhebliche Unschärfen bestehen (was in erster Linie auf die in diesem Bereich relativ ungenaue Berufsklassifikation des SAMS 98 zurückzuführen ist). Ich beschränke mich deshalb auf die Analyse des persönlichen Erwerbseinkommens. Das erzielte Einkommen ist sicherlich einer der wichtigsten Aspekte beruflichen Erfolgs und erlaubt zudem eine detaillierte Differenzierung. Nicht zuletzt besteht der Vorteil einer vergleichsweise einfachen Mes-

gadier), Stabsoffiziere (Oberst, Oberstleutnant, Major), Hauptmann, Subalternoffiziere (Oberleutnant, Leutnant). sung. Als Grundlage für die Analysen dient das folgende, aus der Humankapitaltheorie abgeleitete Regressionsmodell (vgl. Mincer 1974; zusammenfassend auch Franz 1996, Willis 1992):

$$
\ln w=\beta_{0}+\beta_{1} s+\beta_{2} x+\beta_{3} x^{2}+\varepsilon
$$

Der logarithmierte Stundenlohn $w$ wird in Abhängigkeit von der Schulbildung $s$ sowie der Berufserfahrung $x$ gesetzt. Um dem Phänomen abnehmender Zuwachsraten gerecht zu werden, wird der Einfluss der Berufserfahrung parabolisch modelliert. Durch die Logarithmierung der abhängigen Variablen lassen sich die Koeffizienten näherungsweise als Prozenteffekte auf den Stundenlohn interpretieren. Eine Erhöhung der Schulbildung um eine Einheit würde also mit einer Veränderung des erwarteten Stundenlohnes um ca. $\beta_{1} \cdot 100$ Prozent einhergehen (genauer: $\left[\exp \beta_{1}-1\right] \cdot 100$ Prozent).

Als Ausgangsgröße zur Bildung der abhängigen Variablen $w$ (Stundenlohn) dient das von den Befragten angegebene monatliche Nettoeinkommen, ${ }^{5}$ welches durch 3.64-mal die wöchentlichen Arbeitsstunden geteilt wird. ${ }^{6}$ Als wöchentliche Arbeits-

\footnotetext{
${ }^{5}$ Das persönliche Monatseinkommen abzüglich Sozialversicherungsbeiträge, aber vor Abzug der Steuern. Zu bemerken ist, dass es sich beim Nettoeinkommen bereits um eine konstruierte Variable handelt, da ein Teil der Befragten die Einkommenshöhe in anderer Form angegeben hat (z. B. monatliches Bruttoeinkommen, jährliches Nettoeinkommen, etc.). Zur genauen Bildung der Variable vgl. Diekmann et al. 1999.

${ }^{6}$ Der Faktor 3.64 ergibt sich aus der Anzahl Wochen pro
} 
stunden werden die Angaben zu den gewöhnlich pro Woche für den Beruf gearbeiteten Stunden verwendet, bei Fehlen dieser Angaben die vertraglich vereinbarten Wochenarbeitsstunden.

Die Schulbildung wird aus den Angaben der Befragten über ihren höchsten Ausbildungsabschluss abgeleitet (Bildungsjahre). ${ }^{7}$ Die Berechnung der Anzahl der Jahre Berufserfahrung erfolgt nach der Formel „Alter - Bildungsjahre - 6.5“. Bei dieser aus der Literatur bekannten Schätzung (z. B. Mincer 1974) wird vereinfachend angenommen, dass alle Befragten nach Abschluss der Ausbildung durchgehend erwerbstätig waren (was, solange nur Männer betrachtet werden, zu keinen größeren Verzerrungen führen sollte).

Der Einfluss des militärischen Rangs wird schließlich durch Aufnahme weiterer additiver Komponenten in die Humankapitalgleichung analysiert. Die entsprechenden Variablen werden weiter unten erläutert.

\section{Ergebnisse}

Bevor ich mich den Ergebnissen der Regressionsschätzungen zuwende, sollen die Befragten selbst über den subjektiv wahrgenommenen Einfluss der militärischen Position auf die zivile Karriere berichten (Tabelle 2). ${ }^{8}$ Im Widerspruch zur verbreiteten Meinung des Nutzens der militärischen Karriere gibt die große Mehrheit der Befragten an, dass die militärische Position überhaupt keinen Einfluss auf die zivile Berufskarriere hatte. Werden die Ergeb-

Monat (4.3), einem 13. Monatslohn und einem Monat bezahlten Urlaubs pro Jahr. Der Faktor ist hier mangels genauerer Angaben zu Ferientagen und Anzahl Monatslöhnen konstant und hat somit nur kosmetischen Charakter (für unsere Zwecke würde es auch reichen, den Monatslohn nur durch die Wochenarbeitsstunden zu teilen).

${ }^{7}$ Gemäß der folgenden Zuordnung (gestützt auf die Beschreibung der Ausbildungsgänge in Bundesamt für Statistik 1999a, 1999b): ohne Abschluss 8 Jahre; obligatorische Schule 9 Jahre; Anlehre 9.75 Jahre; Handelsschule/Haushaltslehrjahr 10 Jahre; Berufslehre 10.5 Jahre; Diplommittelschule 11.5 Jahre; Vollzeitberufsschule, Berufsmaturität, Meisterdiplom/Fachausweis 12 Jahre; gymnasiale Maturität, Techniker-/Fachschule 12.5 Jahre; höhere Fachschule/HTL/HWV 15 Jahre; Uni/Hochschule 17.5 Jahre; andere Ausbildungen 9 Jahre.

${ }^{8}$ Die genaue Frage lautete: „Was meinen Sie, hat Ihre militärische Position Ihre berufliche Karriere stark begünstigt, begünstigt, teils/teils, behindert oder stark behindert oder hatte sie gar keinen Einfluss? “ Die Kategorien „stark begünstigt“ und „begünstigt“ sowie „stark behindert“ und „behindert" wurden für die Analyse zusammengefasst. nisse jedoch nach militärischen Positionen der Befragten differenziert, so zeigt sich ein deutlicher, mit der These des positiven Effekts der militärischen Karriere in Einklang stehender Zusammenhang: Mit zunehmendem militärischen Rang steigt der Anteil der Befragten, die von einem begünstigenden Einfluss berichten, stark an. So beträgt dieser Anteil in der Kategorie der Hauptleute und Stabsoffiziere fast $40 \%$ (gegenüber $5 \%$ in der Kategorie der Soldaten). Die Frage ist hier natürlich, inwieweit dieser Zusammenhang in messbaren Größen des Berufserfolgs eine Entsprechung findet. Zudem informieren die Resultate nicht über die Gründe der positiven Beeinflussung.

Da zur Schätzung der Regressionsmodelle ein persönliches Erwerbseinkommen vorhanden sein muss, beschränke ich die Stichprobe in der Folge auf erwerbstätige Personen. Zudem schließe ich beruflich Selbständige, Lehrlinge sowie noch nicht ausgehobene (rekrutierte) Personen als selektive und nur schwer vergleichbare Gruppen von den Analysen aus. ${ }^{9}$ Tabelle 3 gibt eine erste bivariate Übersicht über die durchschnittliche Einkommenshöhe nach militärischem Rang. Während zwischen Personen, die keinen Militärdienst leisten (bzw. geleistet haben), Soldaten und Unteroffizieren kaum gravierende Unterschiede bestehen, heben sich die Leutnants und besonders die Hauptleute und Stabsoffiziere deutlich $a b$. So verfügen die Leutnants im Schnitt über ein um $28 \%$ höheres Monatseinkommen als die Soldaten (bzw. einen um $18 \%$ höheren Stundenlohn) und die Hauptmänner und Stabsoffiziere gar über ein um $85 \%$ höheres Einkommen (bzw. einen um gut $50 \%$ höheren Stundenlohn). ${ }^{10}$

Da nicht-experimentelle Daten vorliegen, darf kaum davon ausgegangen werden, dass die Unterschiede in den Löhnen tatsächlich in diesem Umfang auf den Einfluss des militärischen Ranges zurückzuführen sind. Bekanntermaßen ist mit zunehmendem Alter (bzw. zunehmender Berufserfahrung) ein höheres Einkommen zu erwarten, aber auch der militärische Rang ist vom Alter abhängig. Zudem sind sowohl Einkommen wie militärische Ränge mit Bildung assoziiert. Um die Einflüsse von Berufserfahrung und Bildung zu kontrollieren, wird

\footnotetext{
9 Weiterhin entfallen 94 Fälle mit unbekannten Werten für mindestens eine der im Folgenden verwendeten Variablen. Es handelt sich zur Hauptsache um Fälle mit fehlenden Einkommensangaben.

${ }^{10}$ Die relativen Unterschiede treten beim Monatseinkommen deutlicher zu Tage als bei den Stundenlöhnen, weil Personen mit hohem Stundenlohn tendenziell mehr Stunden pro Woche arbeiten.
} 
Tabelle 2 Selbst berichteter Einfluss der militärischen Position auf die zivile berufliche Karriere (Spaltenprozente)

\begin{tabular}{lccccc}
\hline Einfluss auf Karriere & Soldat/Hilfsdienst & Unteroffizier & (Ober-)Leutnant & Hauptmann oder höher & Total \\
\hline Begünstigt & 5 & 14 & 20 & 39 & 9 \\
Teils/teils & 4 & 10 & 29 & 23 & 7 \\
Behindert & 8 & 4 & 2 & 3 & 7 \\
Kein Einfluss & 82 & 72 & 49 & 35 & 77 \\
\hline Total & 100 & 100 & 100 & 100 & 100 \\
\hline
\end{tabular}

Anmerkungen: Männer mit Schweizer Staatsbürgerschaft im Alter von 18-70 Jahren, die Militärdienst leiten bzw. geleistet haben; $N=940 ; \chi^{2}(9)=123.6, p=0.000 ;$ Quelle: SAMS 98, ungewichtet.

Tabelle 3 Monatseinkommen und Stundenlohn nach militärischem Rang

\begin{tabular}{lccccc}
\hline & \multicolumn{2}{c}{ Monatsnettoeinkommen } & \multicolumn{2}{c}{ Netto-Stundenlohn } & \\
& Mittel & Median & Mittel & Median & N \\
\hline Kein Militärdienst & 5384 & 5000 & 32.8 & 29.5 & 123 \\
Soldat/Hilfsdienst & 5606 & 5200 & 34.5 & 32.5 & 424 \\
Unteroffizier & 5739 & 5200 & 35.9 & 33.2 & 112 \\
(Ober-)Leutnant & 7178 & 7200 & 40.6 & 39.6 & 25 \\
Hauptmann oder höher & 10367 & 10500 & 52.3 & 54.9 & 18 \\
\hline Total & 5767 & 5200 & 35.1 & 32.7 & 702 \\
\hline
\end{tabular}

Anmerkungen: Abhängig erwerbstätige Männer mit Schweizer Staatsbürgerschaft im Alter von 18-70 Jahren, ohne Lehrlinge und noch nicht Ausgehobene, zudem Ausschluss von Fällen mit fehlenden Werten für mindestens eine der in den nachfolgenden Regressionsmodellen verwendeten Variablen; Mittel: arithmetisches Mittel; Quelle: SAMS 98, ungewichtet.

im Folgenden die aus der Humankapitaltheorie abgeleitete Regressionsgleichung verwendet. Die Tabellen 4, 5 und 6 zeigen die Ergebnisse. ${ }^{11} \mathrm{Im}$ Anhang befinden sich zudem deskriptive Statistiken zu den verwendeten Variablen.

Modell 1 in Tabelle 4 repliziert den bivariaten $\mathrm{Zu}$ sammenhang aus Tabelle 3, wobei hier vereinfachend und aufgrund der geringen Fallzahlen bei

11 Obwohl sich die Auswahlwahrscheinlichkeiten der einzelnen Zielpersonen des SAMS 98 durch den Stichprobenplan bedingt je nach Haushaltsgröße unterscheiden (vgl. Diekmann et al. 1999: 8f.), verzichte ich bei der Schätzung der Regressionsmodelle auf eine entsprechende Gewichtung der Daten. Wie Winship und Radbill (1994) zeigen, sind ungewichtete Regressionsschätzungen i.d.R. konsistent und unverzerrt und aufgrund höherer Effizienz einer gewichteten Schätzung vorzuziehen (die Standardfehler bei gewichteten Modellen können nach der Methode von White 1980 konsistent geschätzt werden, womit aber ein Effizienzverlust einhergeht). Ob eine Gewichtung trotzdem angezeigt ist (d.h., ob die ungewichtete Schätzung verzerrt ist), kann mit einer einfachen Methode nach DuMouchel und Duncan (1983) getestet werden. Die Anwendung dieses Tests liefert im vorliegenden Fall keine Hinweise auf die Notwendigkeit einer Gewichtung. den militärischen Rängen nur noch zwischen Soldaten bzw. Hilfsdienstleistenden, Unteroffizieren und Offizieren unterschieden wird. Da der Effekt des militärischen Ranges im Zentrum des Interesses steht, weniger aber ein Effekt des Militärdienstes an sich, werden Personen, die keinen Militärdienst leisten bzw. geleistet haben, vorerst ausgeschlossen. Verglichen mit der Referenzgruppe der Soldaten verfügen die Offiziere gemäß Modell 1 über einen fast 30\% höheren Stundenlohn. Werden nun Bildung und Berufserfahrung kontrolliert (Modell 2), so schrumpft der „Offizierseffekt“ auf rund $12 \%$. Er ist aber nach wie vor signifikant. Auch unter standardmäßiger Kontrolle von Humankapital erzielen also die Offiziere einen höheren Stundenlohn als die Soldaten. Die Effekte von Bildung (Rendite von gut $5 \%$ pro Jahr) und Berufserfahrung (abnehmende Rendite von anfänglich gut $4 \%$ pro Jahr; maximaler Stundenlohn bei rund 34 Jahren Berufserfahrung) liegen dabei im Rahmen der Erfahrungswerte für Schweizer Männer (vgl. z.B. Kugler 1988, Diekmann/Engelhardt 1995, Bonjour 1997, Henneberger/Sousa-Poza 1999, Falter/Ferro Luzzi 2000, Franzen 2001).

Die Hypothese, dass Personen in höheren militärischen Rängen beruflich erfolgreicher sind bzw. bes- 
Tabelle 4 Einkommensregressionen (OLS, $t$-Werte in Klammern)

\begin{tabular}{|c|c|c|c|c|}
\hline & Modell 1 & Modell 2 & Modell 3 & Modell 4 \\
\hline \multicolumn{5}{|c|}{ Militärischer Rang (RG: Soldat): } \\
\hline \multirow[t]{2}{*}{ - Unteroffizier } & 0.037 & 0.026 & 0.025 & 0.025 \\
\hline & $(0.96)$ & $(0.83)$ & $(0.79)$ & $(0.80)$ \\
\hline \multirow[t]{2}{*}{ - Offizier } & $0.258^{* * *}$ & $0.117^{*}$ & $0.111^{*}$ & $0.111^{*}$ \\
\hline & $(4.46)$ & $(2.38)$ & $(2.26)$ & $(2.25)$ \\
\hline \multirow[t]{2}{*}{ Bildungsjahre } & & $0.054^{* * *}$ & $0.054^{* * *}$ & $0.054^{* * *}$ \\
\hline & & $(10.03)$ & $(9.99)$ & (9.99) \\
\hline \multirow[t]{2}{*}{ Berufserfahrung } & & $0.046^{* * *}$ & $0.046^{* * *}$ & $0.045^{* * *}$ \\
\hline & & $(10.21)$ & $(10.22)$ & $(10.00)$ \\
\hline \multirow[t]{2}{*}{ Berufserfahrung $2 / 100$} & & $-0.068^{* * *}$ & $-0.068^{* * *}$ & $-0.067^{* * *}$ \\
\hline & & $(-7.24)$ & $(-7.27)$ & $(-7.08)$ \\
\hline
\end{tabular}

Arbeitsanstrengung

Führungsposition

Konstante

$3.482^{* * *}$

$2.265^{* * *}$

Konstante

(198.09)

(27.89)

$2.257^{* * *}$

$2.253^{* * *}$

\begin{tabular}{lllll}
\hline Korrigiertes $R^{2}$ & 0.030 & 0.350 & 0.351 & 0.350
\end{tabular}

(27.72)

(27.51)

Anmerkungen: Abhängig erwerbstätige Männer mit Schweizer Staatsbürgerschaft im Alter von 18-70 Jahren, die Militärdienst leisten bzw. geleistet haben, ohne Lehrlinge; $N=579 ;$ Abhängige Variable: logarithmierter Netto-Stundenlohn; RG: Referenzgruppe; ${ }^{+} p<0.1$, ${ }^{*} p<0.05,{ }^{* *} p<0.01,{ }^{* * *} p<0.001$ (zweiseitig); Quelle: SAMS 98, ungewichtet.

ser bezahlte Arbeit ausüben, scheint sich zu bewähren. ${ }^{12}$ Aber wie kann dieser Effekt erklärt werden? In den Modellen 3 und 4 in Tabelle 4 wird getestet, ob Selbstselektion oder Investitionen in das $\mathrm{Hu}$ mankapital (Führungskompetenzen) zu dem Einkommensbonus führen. Zur Prüfung der Selbstselektionshypothese wird ein Indikator verwendet, der über das Ausmaß an Arbeitsanstrengung Auskunft gibt. ${ }^{13}$ Wenn man argumentiert, dass Personen aus

\footnotetext{
12 Wobei man der Prüfung der Hypothese natürlich noch mit weiteren Instrumenten wie etwa der Propensity-ScoreMethode nachgehen könnte; vgl. zusammenfassend Winship/Morgan 1999.

${ }^{13}$ Die entsprechende Frage lautete: „Bemühen Sie sich im Beruf mehr zu leisten als das, was von Ihnen gefordert wird? “ mit den Antwortkategorien „nein“, „ja, nur wenig “, ,ja, einiges“ und „ja, viel“. Aufgrund der schiefen Verteilung der Antworten fasse ich die ersten drei Kategorien zusammen. Der Indikator der Arbeitsanstrengung nimmt also den Wert 1 an für Personen, die viel mehr als
}

dem gleichen Grund beruflich erfolgreicher sind, wie sie in höhere militärische Ränge vorstoßen, nämlich weil ihnen eine gelungene Karriere im Militär wie auch Beruf wichtig ist und sie sich deshalb in beiden Gebieten mehr anstrengen als andere, müsste der Offizierseffekt unter Berücksichtigung dieses Indikators verschwinden. Wie man in Modell 3 sieht, ist dies nicht der Fall: Der Offizierseffekt bleibt bei rund $12 \%$ bestehen. Zugegebenermaßen ist der Indikator für die Arbeitsanstrengung in dem Modell nicht besonders erklärungskräftig, was Zweifel an der Validität des Maßes aufkommen lassen mag. Der Effekt weist zwar in die erwartete Richtung, ist aber nicht signifikant.

$\mathrm{Zu}$ einem ähnlichen Schluss kommt man bei der Betrachtung von Modell 4 (Tabelle 4): Auch wenn berücksichtigt wird, ob eine Person im Erwerbsleben

gefordert zu leisten angegeben haben, und ist sonst gleich 0 . 
eine Führungsposition einnimmt, bleibt der Offizierseffekt praktisch unberührt erhalten. Er kommt also offenbar nicht dadurch zustande, dass Personen aufgrund der militärischen Führungsausbildung eher in Führungspositionen gelangen und diese (unter Kontrolle von Bildung und Berufserfahrung) besser entlohnt werden als andere Arbeitsstellen. ${ }^{14}$

Es bleibt die dritte Hypothese zu testen, die besagt, dass Personen in höheren militärischen Rängen aufgrund des militärischen sozialen Beziehungsnetzes zu besser bezahlten Jobs gelangen. Dazu soll ein Indikator in das Modell aufgenommen werden, der angibt, ob die aktuelle Stelle über soziale Kontakte gefunden wurde (gegenüber der Stellenfindung via formelle Wege). ${ }^{15}$ Die Ergebnisse sind in Modell 5 (Tabelle 5) dargestellt und zeigen deutlich, dass auch die Stellenfindung über soziale Kontakte den Offizierseffekt nicht zu erklären vermag. Hält man sich an Mark Granovetters These der „Stärke der schwachen Beziehungen“ (1973), würde man das auch nicht zwingend erwarten. Nach Granovetter (1973,1974, 1983; vgl. auch Liu/Duff 1972) sind es nämlich nicht unbedingt soziale Kontakte allgemein, die sich günstig auswirken, sondern besonders die „schwachen“ Beziehungen („weak ties“). Denn Personen, zu denen man eine eher lose Beziehung pflegt, verbinden häufig unterschiedliche soziale Welten und machen dadurch neue Informationsquellen (z.B. über offene Arbeitsstellen) zugänglich (vgl. auch Feld 1997). Beziehungen dieser Art werden i.d.R. als „bridging ties“ bezeichnet. Aufgrund der Organisationsstruktur der Armee sollten solche Beziehungsmuster gerade in der Führungsriege des Militärs verstärkt vorliegen. Auf den untersten hierarchischen Stufen richtet sich die Armeeeinteilung grundsätzlich nach dem Jugendwohnort, was eine Durchmischung von Personen aus sozial fernen Netzwerken zumindest behindert.

\footnotetext{
${ }^{14}$ Der Indikator wurde aus der Frage „Gehört es zu Ihren Aufgaben, andere Mitarbeiter anzuleiten oder ihre Arbeit zu beaufsichtigen?" abgeleitet, die mit „ja“ oder „nein“ beantwortet werden konnte.

${ }^{15}$ Die entsprechende Frage lautete: „Wie genau sind Sie auf Ihren Arbeitsplatz gestoßen? “ Die Antworten „fragte eine mir bekannte Person“, „wurde von einer mir bekannten Person darauf aufmerksam gemacht, die wusste, dass ich Arbeit suche“, „wurde von einer mir bekannten Person darauf aufmerksam gemacht, die nicht wusste, dass ich Arbeit suche“ und „durch eine mir bis dahin unbekannte Person" werte ich als Stellenfindung über soziale Kontakte, die Antworten „Anzeige in Zeitung “, „Arbeitsamt“, „Stellenvermittlungsfirma“, „Head Hunter“, „direkt bei Unternehmen beworben“ und „anderes“ als Stellenfindung über formelle Wege.
}

Mit dem Aufstieg in höhere Ränge nimmt die Reichweite der militärischen Kontakte (d.h. die Anzahl bridge-artiger Verbindungen) insbesondere in Form von Beziehungen zu hierarchisch Gleichgestellten jedoch kontinuierlich zu. Zudem schafft die hierarchische Abgrenzung nach unten eine gemeinsame Identität. Das persönliche multiplexe Netzwerk aus Freundschafts-, Arbeits- und sonstigen Beziehungen wird mit höherem militärischem Rang also zunehmend um die für andere Personen nicht zugängliche Ebene der militärischen Kontakte erweitert. Diese Beziehungen sind, zumindest was die Kontakthäufigkeit und die Überschneidung mit den zivilen Netzwerken anbelangt, i.d.R. als „schwache“ Beziehungen zu bezeichnen (vgl. Oester 1996). Sie werden aber - so die zentrale Besonderheit des Milizsystems in der Schweiz - während der Dienstpflicht bis in ein relativ hohes Alter regelmäßig aufgefrischt. Hinzu kommen zum Teil auch private Zusammenkünfte bei Veranstaltungen der Offiziersgesellschaften.

Für die weitere Analyse soll daher zwischen der Stellenfindung über „starke“ versus „schwache“ soziale Kontakte unterschieden werden, um zu ermitteln, ob sich der Offizierseffekt durch häufigeren Rückgriff auf schwache soziale Kontakte erklären lässt. ${ }^{16}$ Die Ergebnisse (Modell 6 in Tabelle 5) fallen auch hier negativ aus. Nicht nur dass der Offizierseffekt unberührt erhalten bleibt, die Effekte der sozialen Kontakte weisen sogar tendenziell in eine den Erwartungen entgegengesetzte Richtung: Die Stellenfindung über schwache soziale Kontakte wirkt sich verglichen mit der Stellenfindung über formelle Wege eher negativ auf den Stundenlohn aus (nicht signifikant). Das Ergebnis, dass Personen in höheren militärischen Positionen im zivilen Beruf einen höheren Lohn erzielen, kann demnach auch

${ }^{16}$ Den Personen, die angaben, ihre Arbeitsstelle über soziale Kontakte gefunden zu haben, wurde zusätzlich die Frage „Wie intensiv war Ihr Kontakt zu der Person zum Zeitpunkt Ihrer Stellensuche? “ gestellt (mit geringfügigen Modifikationen übernommen von Preisendörfer/Voss 1988). Ich werte die Antworten „wir sahen uns oft und regelmäßig“ und ,wir hatten recht oft und regelmäßig brieflichen oder telefonischen Kontakt", als starke Kontakte, die Antworten ,wir trafen uns nur bei bestimmten Gelegenheiten oder hatten gelegentlich brieflichen oder telefonischen Kontakt“, „wir trafen uns zufällig“, „wir kannten und kaum bzw. gar nicht und wurden durch gemeinsame bekannte, dritte Personen zusammengebracht" und „nichts trifft zu“ als schwache Kontakte. Dies ist zugegebenermaßen eine etwas vereinfachende Kategorisierung, die sich in erster Linie auf die Häufigkeit des Kontaktes stützt. Zu Vorschlägen für differenziertere Operationalisierungen vgl. etwa Marsden und Campbell 1984. 
Tabelle 5 Einkommensregressionen (Fortsetzung; $t$-Werte in Klammern)

\begin{tabular}{|c|c|c|c|c|}
\hline & Modell 5 & Modell 6 & Modell 7 & Modell 8 \\
\hline \multicolumn{5}{|l|}{ Militärischer Rang (RG: Soldat): } \\
\hline \multirow{2}{*}{ - Unteroffizier } & 0.026 & 0.022 & 0.021 & 0.019 \\
\hline & $(0.84)$ & $(0.69)$ & $(0.67)$ & $(0.61)$ \\
\hline \multirow[t]{2}{*}{ - Offizier } & $0.117^{*}$ & $0.117^{*}$ & 0.064 & 0.056 \\
\hline & $(2.37)$ & $(2.39)$ & $(1.18)$ & $(1.01)$ \\
\hline \multirow[t]{2}{*}{ Bildungsjahre } & $0.054^{* * *}$ & $0.055^{* * *}$ & $0.056^{* * *}$ & $0.055^{* * *}$ \\
\hline & $(9.99)$ & $(10.19)$ & $(10.25)$ & $(10.18)$ \\
\hline \multirow[t]{2}{*}{ Berufserfahrung } & $0.046^{* * *}$ & $0.047^{* * *}$ & $0.046^{* * *}$ & $0.046^{* * *}$ \\
\hline & $(10.20)$ & $(10.37)$ & $(10.40)$ & $(10.22)$ \\
\hline \multirow[t]{2}{*}{ Berufserfahrung $2 / 100$} & $-0.068^{* * *}$ & $-0.069^{* * *}$ & $-0.069^{* * *}$ & $-0.068^{* * *}$ \\
\hline & $(-7.23)$ & $(-7.37)$ & $(-7.36)$ & $(-7.25)$ \\
\hline \multirow[t]{2}{*}{ Arbeitsanstrengung } & & & & 0.030 \\
\hline & & & & $(1.17)$ \\
\hline \multirow[t]{2}{*}{ Führungsposition } & & & & 0.024 \\
\hline & & & & $(0.90)$ \\
\hline \multirow[t]{2}{*}{ Stellenfindung: soziale Kontakte } & -0.007 & & & \\
\hline & $(-0.25)$ & & & \\
\hline \multicolumn{5}{|l|}{ Stellenfindung (RG: formell): } \\
\hline \multirow[t]{2}{*}{ - starke soziale Kontakte } & & 0.044 & 0.040 & 0.040 \\
\hline & & $(1.23)$ & $(1.12)$ & $(1.11)$ \\
\hline \multirow[t]{2}{*}{ - schwache soziale Kontakte } & & $-0.079^{+}$ & $-0.115^{* *}$ & $-0.116^{* *}$ \\
\hline & & $(-1.92)$ & $(-2.68)$ & $(-2.69)$ \\
\hline \multirow[t]{2}{*}{ Starke Kontakte* Offizier } & & & 0.036 & 0.047 \\
\hline & & & $(0.23)$ & $(0.29)$ \\
\hline \multirow[t]{2}{*}{ Schwache Kontakte* Offizier } & & & $0.423^{* *}$ & $0.407^{* *}$ \\
\hline & & & $(2.88)$ & $(2.76)$ \\
\hline \multirow[t]{2}{*}{ Konstante } & $2.268^{* * *}$ & $2.248^{* * *}$ & $2.250^{* * *}$ & $2.232^{* * *}$ \\
\hline & $(27.63)$ & $(27.37)$ & $(27.50)$ & $(26.99)$ \\
\hline Korrigiertes $R^{2}$ & 0.349 & 0.354 & 0.361 & 0.362 \\
\hline
\end{tabular}

Anmerkungen: Abhängig erwerbstätige Männer mit Schweizer Staatsbürgerschaft im Alter von 18-70 Jahren, die Militärdienst leisten bzw. geleistet haben, ohne Lehrlinge; $N=579 ;$ Abhängige Variable: logarithmierter Netto-Stundenlohn; RG: Referenzgruppe; ${ }^{+} p<0.1$, ${ }^{*} p<0.05,{ }^{* *} p<0.01,{ }^{* * *} p<0.001$ (zweiseitig); Quelle: SAMS 98, ungewichtet.

nicht mit Granovetters Theorie der Stärke der „weak ties“ erklärt werden.

Zieht man allerdings einige neuere Überlegungen und Befunde zu Granovetters Ansatz in Betracht, muss von einem etwas komplexeren Zusammen- hangsmuster ausgegangen werden. Verschiedene Autoren weisen darauf hin, dass der Nutzen sozialer Kontakte - seien es nun schwache oder starke von Rahmenbedingungen wie der Netzwerkstruktur (Montgomery 1991, 1992, Podolny/Baron 
1997, Flap/Völker 2001), der eigenen sozialen Position im Netzwerk (Lin et al. 1981a, Wegener 1987, 1989, 1991) bzw. der sozialen Position der Kontaktperson (Lin et al. 1981a, 1981b, de Graaf/Flap 1988) abhängt. So zeigt Wegener (1991), dass sich die Stellenfindung über schwache soziale Kontakte vor allem für Personen in beruflichen Positionen mit hohem Prestigewert bezahlt macht, während Personen in tieferen beruflichen Positionen eher von starken sozialen Kontakten profitieren können. Kurz: es besteht ein Interaktionseffekt zwischen der eigenen Position und der Wirkung sozialer Kontakte. Die Erklärung hierfür stützt sich auf die Überlegung, dass vor allem Kontakte zu statushöheren Personen für die Stellenfindung nützlich sind und dass auch Netzwerke starker Beziehungen zu einem gewissen Grad heterogen sein können bezüglich des Status ihrer Mitglieder (vgl. Wegener 1991). Nach der Standardtheorie der Stärke der schwachen Beziehungen können Verbindungen zu statushöheren Personen hauptsächlich als schwache soziale Kontakte realisiert werden („,bridging ties“ zu statushöheren Netzwerken). Ein positiver Effekt auf die berufliche Mobilität wird daher nur von den schwachen sozialen Kontakten erwartet. Lässt man jedoch Statusheterogenität innerhalb der Netzwerke starker Beziehungen zu, so erweisen sich für Personen, die innerhalb eines solchen Netzwerks einen relativ niedrigen Status einnehmen, auch starke soziale Kontakte als nützlich. Nur die Personen, die sich eher am oberen Ende der Hierarchie befinden, sind mangels starker Kontakte zu statushöheren Personen im eigenen Netzwerk zu einer Orientierung nach außen gezwungen.

Übertragen auf den hier zu erklärenden Sachverhalt lassen sich zum Teil ähnliche Überlegungen anstellen. Aus der Annahme einer traditionellen Verflechtung der militärischen Elite mit anderen gesellschaftlichen Eliten z.B. in Wirtschaft, Politik und Verwaltung folgt, dass Männer, wenn sie in höhere militärische Ränge vorstoßen, ihr Netzwerk mit sozialen Verbindungen zu Personen anreichern können, die in verschiedener Hinsicht einen hohen Status aufweisen. Es wird also der Zugang zu einer Gruppe an gesellschaftlich vorteilhaft positionierten Personen hergestellt, die sich als besonders geeignete Informationsträger im Rahmen der Stellensuche erweisen können. Männer in unteren militärischen Rängen oder Personen, die keinen Militärdienst leisten, mögen vielleicht in ähnlichem Ausmaß über schwache soziale Kontakte verfügen. Die Qualität dieser Kontakte wird sich aber insofern unterscheiden, als eine tendenziell geringere Konzentration an statushohen Kontaktpersonen vorliegt. Zu erwarten ist somit ein Interaktionseffekt zwischen der militärischen Position und der Wirkung sozialer Kontakte: Die Stellenfindung über schwache soziale Kontakte sollte sich besonders für Personen in höheren militärischen Rängen bezahlt machen. ${ }^{17}$

Zusammenfassend nochmals die für die Sozialkapitalhypothese relevanten Argumente: (1) Die Teilnahme am Militärdienst schafft zusätzliche schwache soziale Beziehungen, die unterschiedliche soziale Welten verbinden und den Zugriff auf alternative Informationsquellen ermöglichen. (2) Die militärischen Kontakte werden aufgrund der langen Wehrpflicht über eine bedeutende Dauer des zivilen Erwerbslebens aufrechterhalten. (3) Aus strukturellen Gründen nimmt die Möglichkeit zur Schaffung solcher Beziehungen mit höheren militärischen Rängen zu: Die Reichweite und der (informelle) Organisationsgrad der Kontakte steigt. Auch erhöhen sich die Dauer der formellen Aufrechterhaltung sowie die Häufigkeit der Kontakte durch die zeitliche Ausweitung der Wehrpflicht und die zusätzlichen Diensttage. (4) Mit dem militärischen Rang nimmt die Kontaktdichte zu Personen mit hohem Status in den außermilitärischen Sozialbereichen wie Wirtschaft, Politik oder Verwaltung zu. Der Aufstieg in höhere militärische Positionen ermöglicht somit den Zugang zu einer gesellschaftlichen Elite, zu Personen also, die sich für die eigene berufliche Promotion als besonders nützlich erweisen können. Aus den Punkten (1) bis (3) folgt, dass Personen in höheren militärischen Positionen i.d.R. über ein ausgeprägtes Netzwerk an schwachen sozialen Kontakten verfügen, was, in Anlehnung an Granovetters These von der Stärke der schwachen Beziehungen, ihren größeren beruflichen Erfolg erklären könnte. Wie erläutert, wird die Wirkung schwacher sozialer Kontakte allerdings kaum per se vorteilhaftig sein, sondern von weiteren Bedingungen wie etwa dem beruflichen Status der Kontaktperson abhängen. Gemäß Punkt (4) erfüllt das Beziehungsnetz der militärischen Führungskräfte diese Bedingungen in besonderem Maße, was einen positiven Interaktionseffekt zwischen dem militärischen Rang und der Wirkung schwacher sozialer Kontakte erwarten lässt.

\footnotetext{
${ }^{17}$ Diese Erklärung mag auf den ersten Blick zirkulär erscheinen. $\mathrm{Zu}$ bedenken ist allerdings, dass die Verflechtung der militärischen mit anderen gesellschaftlichen Eliten organisatorisch (und historisch) bedingt sein kann. Zudem wird die Konzentration an statushohen Personen in militärischen Führungsrängen auf weiteren Zusammenhängen wie z.B. dem Einfluss von Bildung beruhen. Der erläuterte Netzwerkeffekt darf höchstens als Verstärkereffekt gesehen werden.
} 
Von Interesse erscheint an dieser Stelle der Hinweis, dass die Unterscheidung in starke und schwache Kontakte für die theoretische Argumentation nicht unbedingt zentral ist. Die Unterscheidung ist hier hauptsächlich durch die spezifische Operationalisierung der Beziehungsstärke als Kontakthäufigkeit bedingt, denn in dieser Betrachtungsweise sind die militärischen Kontakte i.d.R. als schwache Kontakte zu bezeichnen. Der theoretisch wichtige Aspekt ist vielmehr, ob die Beziehungen unterschiedliche soziale Welten verbinden, was zwar für schwache soziale Kontakte und insbesondere für die Beziehungen unter militärischen Führungspersonen mit hoher Wahrscheinlich zutrifft, u.U. aber auch für starke soziale Kontakte der Fall sein kann. Tatsächlich gibt es Hinweise, dass gerade die Beziehungen zwischen höherrangigen Militärs häufig als starke Verbindungen $\mathrm{zu}$ charakterisieren sind, wenn als Dimension der Beziehungsstärke z. B. das gegenseitige Vertrauen in Betracht gezogen wird (vgl. Einleitung). Unter der plausiblen Annahme, dass zusätzliches Vertrauen in einer Beziehung bei sonst gleich bleibenden Bedingungen den Informationsfluss begünstigt, ist dies neben dem unter Punkt (4) aufgeführten Argument des hohen Status der Kontaktpersonen eine weitere Begründung für die besondere Wirkungsweise des sozialen Netzwerks unter Männern in höheren militärischen Rängen.

Schwache soziale Kontakte sollten sich also, wenn wir die Kontakthäufigkeit als Maß für die Beziehungsstärke beibehalten, besonders für Personen in höheren militärischen Rängen bezahlt machen, nicht aber unbedingt für andere. Die Ergebnisse der empirischen Prüfung dieser Hypothese fallen nun überaus deutlich aus (Modell 7 in Tabelle 5). Wie erwartet ist die Interaktion zwischen der militärischen Position (Offizier) und der Stellenfindung über schwache Kontakte stark positiv und signifikant. Der Haupteffekt der schwachen Beziehungen ist dabei signifikant negativ. Dies bedeutet, dass sich die Stellenfindung über schwache soziale Kontakte für Nicht-Offiziere eher negativ auf den erzielten Stundenlohn auswirkt, für Offiziere jedoch positiv; der Netto-Effekt für Offiziere ergibt sich aus der Addition der beiden Koeffizienten, also 0.308 , und ist mit $p=0.029$ signifikant von null verschieden. Noch wichtiger erscheint hier allerdings, dass sich in Modell 7 erstmals der Offizierseffekt substantiell verändert: Er fällt von der signifikanten, rund 12-prozentigen Lohnrendite auf deutlich nicht-signifikante $6 \%$. Das heißt, der Einkommensvorteil von Offizieren stellt sich anscheinend nur für diejenigen ein, die mit Hilfe von schwachen sozialen Kontakten zu ihrer aktuellen
Stelle gelangt sind. Der Grund für den Offizierseffekt ist gemäß der Analyse somit im sozialen Beziehungsnetz dieser Personen zu suchen. Das berichtete Zusammenhangsmuster bleibt auch bestehen, wenn die beiden Variablen zur Prüfung der Selbstselektions- und Humankapitalhypothese wieder aufgenommen werden (Modell 8; Netto-Offizierseffekt: $p=0.040$ ).

Gestützt auf die Ergebnisse von Wegener (1991) könnte argumentiert werden, dass der gemessene Netzwerkeffekt nur auftritt, weil die betrachteten Offiziere schon bei ihrer letzten Anstellung eine relativ hohe berufliche Position eingenommen haben und Personen in hohen beruflichen Positionen allgemein von schwachen sozialen Kontakten profitieren. Der Netzwerkeffekt wäre dann nicht eine Funktion des militärischen Ranges, sondern eine Funktion der vor der aktuellen Stelle zuletzt eingenommen beruflichen Position (deren Erreichen sich möglicherweise durch andere Gründe wie etwa Selbstselektion oder Humankapital erklären ließe). Diese Argumentation legt auch eine Ausweitung der betrachteten Population auf alle abhängig erwerbstätigen Männer nahe (also auch auf Personen, die keinen Militärdienst leisten bzw. leisteten). Es soll so geprüft werden, ob sich tatsächlich ein spezieller Netzwerkeffekt nur für die oberen Militärs einstellt oder ob es sich nicht einfach allgemein um die nach Wegener (1991) zu erwartende Abhängigkeit des Vorteils schwacher Beziehungen von der beruflichen Statushöhe handelt.

Die Berechnung eines Modells, das auch Personen, die keinen Militärdienst leisten, berücksichtigt und die Abhängigkeit des Netzwerkeffektes von der letzten beruflichen Stellung modelliert, bestätigt die bisherigen Ergebnisse (Modell 9 in Tabelle 6). Der Effekt der letzten beruflichen Stellung ist zwar insgesamt positiv, und zumindest tendenziell zeigt sich die erwartete positive Interaktion zwischen der letzten beruflichen Stellung und der Stellenfindung über schwache soziale Kontakte; der positive Einfluss schwacher Beziehungen für Offiziere bleibt jedoch uneingeschränkt bestehen (Netto-Effekt: $p=0.037)$. Die Variable der letzten beruflichen Stellung, d.h. der vor der aktuellen Anstellung zuletzt eingenommenen beruflichen Position, unterscheidet dabei zwischen höheren oder leitenden Angestellten einerseits und Arbeitern, einfachen und mittleren Angestellten, beruflich Selbständigen sowie Personen ohne letzte Stelle andererseits. Zu bemerken ist in diesem Zusammenhang, dass auch die Kontrastierung nach weiteren Ausprägungen der letzten Stelle (z.B. zusätzliche Aufnahme eines Indikators für mittlere Angestellte) den Netzwerkeffekt für Of- 
fiziere nicht $\mathrm{zu}$ beeinträchtigen vermag; auf eine Darstellung davon wird hier verzichtet. (Von einer genaueren Status-Messung der letzten beruflichen Position z.B. mit Hilfe von Prestige-Skalen oder dem zuletzt erzielten Einkommen musste mangels geeigneter Daten leider abgesehen werden.) Weiterhin zeigt sich der begünstigende Effekt einer militärischen Karriere auch im Vergleich mit Personen, die keinen Militärdienst leisten (bzw. geleistet haben), zumal diese durchschnittlich sogar ein signifikant geringeres Einkommen aufweisen als die Soldaten.

In Modell 10 (Tabelle 6) wird schließlich noch nach Betriebsgröße und Branche kontrolliert. Diese Variablen beeinflussen bekanntlich das Lohnniveau. Der Einkommensvorteil von Offizieren könnte also nur dadurch zustande kommen, dass sich Offiziere - aus welchen Gründen auch immer - beruflich stärker auf Hochlohnsektoren konzentrieren. Verglichen mit ihren Kollegen aus ähnlichen Betrieben, wären die Offiziere dann nicht mehr als beruflich erfolgreicher zu bezeichnen. Erst eine genauere Analyse der Prozesse, die zur Segregation bezüglich Branche und Betriebsgröße führen, würde Aufschluss darüber geben, ob trotzdem von einem positiven Einfluss des militärischen Rangs auf die zivile Berufskarriere gesprochen werden kann. Entgegen diesen Überlegungen zeigen die Ergebnisse in Tabelle 6 (Modell 10), dass das bisherige Zusammenhangsmuster auch unter Kontrolle dieser Segregation mehr oder weniger erhalten bleibt. ${ }^{18}$ Die Betriebsgröße wie auch die Branche nehmen zwar beide hochsignifikanten Einfluss auf die abhängige Variable (simultaner Test über alle Branchen-Dummies: $p<0.001$ ), für Offiziere besteht aber noch immer ein positiver Netto-Effekt der Stellenfindung über schwache soziale Kontakte $(p=0.036)$.

\section{Diskussion}

Die präsentierten Analysen und Ergebnisse demonstrieren, dass sich bei der Erklärung von sozioökonomischem Status Gedanken über Sozialkapital (insbesondere über Granovetters Ansatz der Stärke der schwachen Beziehungen) als lohnenswert erwei-

\footnotetext{
${ }^{18}$ Die Werte für Betriebsgröße und Branche beruhen auf Selbstauskünften der Befragten. Betriebsgröße: 1 bis 10 kontinuierlich, ab 11 Personen in Klassen (für die Analyse verwendete Werte: 15 für die Antwortvorgabe „11 bis 19 Personen“, 30 für „20 bis 49 Personen“, 75 für „50 bis 99 Personen“ und 150 für „100 und mehr“). Branche: Die ursprüngliche Einteilung in 14 vorgegebene Wirtschaftszweige wurde aus Gründen von z.T. geringen Fallzahlen zu 9 Kategorien zusammengefasst.
}

sen und dies auch trotz teilweise kontroverser Befunde (z. B. Murray et al. 1981, Bridges/Villemez 1986, Marsden/Hurlbert 1988, Preisendörfer/Voss 1988). Zusammenfassend lässt sich festhalten, dass der - in Übereinstimmung mit der allgemeinen Meinung über den zivilen Nutzen einer militärischen Karriere und der durch die Befragten berichteten, subjektiven Wahrnehmung - empirisch anhand einer Querschnittsstichprobe gemessene Einkommensvorteil von Milizoffizieren weder auf Selbstselektion noch auf Investitionen in das Humankapital zurückzuführen ist. Die Resultate deuten vielmehr darauf hin, dass das besondere militärische Beziehungsnetz für den Effekt verantwortlich ist. Es ist anzunehmen, dass der Vorstoß in höhere militärische Ränge den Zugang zu einem wirkungsvollen Kreis an sozialen Kontakten eröffnet, d.h. zu einem Netzwerk von gesellschaftlich - also sozioökonomisch aber z. B. auch politisch - günstig positionierten Männern, die bezüglich der Stellensuche wertvolle Informationen transportieren können und u.U. sogar über Entscheidungsbefugnisse über zu besetzende Positionen verfügen. Die Aktivierung dieser Kontakte bei der Stellensuche führt dann zu der beobachteten besseren Positionierung, und die so aufgestiegenen Personen werden schließlich wiederum zu den Mentoren der nachrückenden Generationen. Am besten kann man sich ein solches Offiziersnetzwerk wohl im Sinne einer „Seilschaft“ (Ambühl 1998) vorstellen, deren Teilnehmer sich abwechselnd bzw. in der Abfolge von Generationen gegenseitig in die Höhe ziehen.

An dieser Interpretation haftet wohlgemerkt teilweise der Charakter einer Spekulation, weil unsere Daten keine Auskunft darüber geben, ob die fraglichen sozialen Kontakte tatsächlich in Zusammenhang mit dem Militärdienst stehen. Geht man von dem Ergebnis aus, dass Personen in höheren militärischen Rängen nur und gerade dann ein höheres Einkommen erzielen, wenn sie ihre aktuelle Stelle über schwache soziale Kontakte gefunden haben, dann fällt es allerdings schwer, eine plausible alternative Erklärung zu finden.

Ein gewichtigeres Problem der vorgelegten Analysen sind die geringen Fallzahlen. So handelt es sich bei den Offizieren, die ihre Stelle über schwache Kontakte gefunden haben, um nicht mehr als eine Handvoll Personen. Für die berichteten Ergebnisse spricht allerdings, dass die Schätzer für die Koeffizienten und Standardfehler auch nach Ausschluss einflussreicher Datenpunkte mehr oder weniger stabil bleiben. ${ }^{19}$ Zudem führt eine alternative

${ }^{19}$ Dies zeigen Replikationen der Modelle unter Aus- 
Tabelle 6 Einkommensregressionen (Fortsetzung)

\begin{tabular}{|c|c|c|c|c|}
\hline & \multicolumn{2}{|c|}{ Modell 9} & \multicolumn{2}{|c|}{ Modell 10} \\
\hline & Koeffizient & $t$-Wert & Koeffizient & $t$-Wert \\
\hline \multicolumn{5}{|l|}{ Militärischer Rang (RG: Soldat): } \\
\hline - kein Militär & $-0.072^{*}$ & -2.32 & $-0.054^{+}$ & -1.80 \\
\hline - Unteroffizier & 0.021 & 0.65 & 0.022 & 0.70 \\
\hline - Offizier & 0.055 & 0.99 & 0.045 & 0.82 \\
\hline Bildungsjahre & $0.052^{* * *}$ & 10.23 & $0.046^{* * *}$ & 9.08 \\
\hline Berufserfahrung & $0.042^{* * *}$ & 10.04 & $0.039^{* * *}$ & 9.56 \\
\hline Berufserfahrung $2 / 100$ & $-0.061^{* * *}$ & -7.01 & $-0.056^{* * *}$ & -6.66 \\
\hline Arbeitsanstrengung & 0.036 & 1.51 & $0.044^{+}$ & 1.91 \\
\hline Führungsposition & 0.029 & 1.12 & 0.040 & 1.61 \\
\hline \multicolumn{5}{|l|}{ Stellenfindung (RG: formell): } \\
\hline - starke soziale Kontakte & -0.006 & -0.19 & 0.008 & 0.24 \\
\hline - schwache soziale Kontakte & $-0.090^{*}$ & -2.18 & -0.059 & -1.47 \\
\hline Starke Kontakte* Offizier & 0.077 & 0.47 & 0.088 & 0.56 \\
\hline Schwache Kontakte ${ }^{*}$ Offizier & $0.391^{* *}$ & 2.62 & $0.351^{*}$ & 2.43 \\
\hline Letzte Stelle: höherer Angestellter & 0.046 & 0.99 & 0.057 & 1.27 \\
\hline Starke Kontakte*höherer Angestellter & 0.035 & 0.33 & 0.036 & 0.36 \\
\hline Schwache Kontakte*höherer Angestellter & 0.217 & 1.48 & 0.213 & 1.51 \\
\hline Betriebsgröße/10 & & & $0.006^{* *}$ & 3.19 \\
\hline \multicolumn{5}{|l|}{ Branche (RG: Industrie): } \\
\hline - Land-/Forstwirtschaft & & & $-0.211^{* *}$ & -2.97 \\
\hline - Baugewerbe & & & -0.009 & -0.22 \\
\hline - Gastgewerbe/Handel/Reparatur & & & $-0.159^{* * *}$ & -3.64 \\
\hline - Verkehr/Nachrichten & & & 0.053 & 0.99 \\
\hline - Banken/Finanzgesellschaften & & & $0.123^{*}$ & 2.33 \\
\hline - Versicherung/Beratung & & & 0.075 & 1.51 \\
\hline - sonstige Dienstleistungen & & & -0.011 & -0.34 \\
\hline - öffentliche Verwaltung & & & $0.089^{*}$ & 2.20 \\
\hline Konstante & $2.309^{* * *}$ & 29.87 & $2.355^{* * *}$ & 30.02 \\
\hline Korrigiertes $R^{2}$ & 0.345 & & 0.391 & \\
\hline
\end{tabular}

Anmerkungen: Abhängig erwerbstätige Männer mit Schweizer Staatsbürgerschaft im Alter von 18-70 Jahren, ohne Lehrlinge und noch nicht Ausgehobene; $N=702$; Abhängige Variable: logarithmierter Netto-Stundenlohn; RG: Referenzgruppe; ${ }^{+} p<0.1, " p<0.05$,

${ }^{* *} p<0.01,{ }^{* * *} p<0.001$ (zweiseitig); Quelle: SAMS 98, ungewichtet.

Modellierung der Einkommensgleichung mit dem logarithmierten Monatseinkommen als abhängiger Variable und den logarithmierten Arbeitsstunden als

schluss von Fällen, die gemäß der Statistik DFBETAS als besonders einflussreich anzusehen sind (als Kriterien für den Ausschluss wurde IDFBETAS| $>2 / \mathrm{N}^{0.5}$ sowie |DFBETASI > 1 verwendet; zum Verfahren siehe Belsley et al. 1980, Bollen/Jackman 1990). Darüber hinaus scheinen auch Multikollinearität und Heteroskedastizität keine Probleme zu bereiten. zusätzlicher Kovariate (vgl. z. B. Brüderl et al. 1993, Diekmann/Engelhardt 1995) zu den gleichen Schlüssen; die Ergebnisse fallen sogar noch etwas deutlicher aus, weil aufgrund des Endogenitätsproblems dieser Modellierung - das Arbeitsangebot hängt der ökonomischen Theorie gemäß vom Lohn ab (vgl. Killingsworth 1983, Bonjour/Gerfin 1995) - die Koeffizienten tendenziell etwas inflationiert werden.

Trotzdem: Die Datenlage ist eher dünn, und es wäre voreilig, einen endgültigen Schluss zu ziehen. Die Resultate sollten möglichst mit weiteren Studien va- 
lidiert werden, die sich erstens auf größere Fallzahlen stützen und zweitens genauere Informationen über die sozialen Netze der Befragten und die Charakteristika der stellenvermittelnden Kontakte enthalten. Auch wäre eine genauere theoretische Modellierung der Wirkungsweise des militärischen Beziehungsnetzwerks - oder verwandter Netzwerke im Allgemeinen - wünschenswert, dies insbesondere auch im Hinblick auf die hier noch nicht angesprochene Forschung zur Geschlechterdiskriminierung auf dem Arbeitsmarkt: Es ist ja durchaus denkbar, dass ein Teil der nach wie vor bestehenden Unterschiede der Arbeitsmarktchancen von Frauen und Männern auf die Funktionstüchtigkeit von Männernetzwerken wie z.B. Studentenverbindungen, Service Clubs oder Offiziersgesellschaften, zurückzuführen ist, die den Zugang zu Personen in Elite-Positionen kontrollieren (vgl. Oester 1996, Ibarra 1992, Moore/White 2001). Hierbei stellt sich weiterhin die Frage, inwieweit die präsentierten Ergebnisse auf andere Länder übertragen werden können. Zwar wird auch etwa in Deutschland die Vorteilhaftigkeit einer Laufbahn als Zeitoffizier für den Zivilberuf hervorgehoben (z. B. Hallerbach
1988); dass aber ein entsprechender Einfluss der in der Armee gebildeten sozialen Netzwerke besteht, darf bis auf weiteres angezweifelt werden. Die lange Dienstzeit bis in ein Alter von 42 bis 52 Jahren und die damit verbundene regelmäßige Auffrischung der sozialen Kontakte sind eben eine Besonderheit des schweizerischen Milizsystems.

Nicht zuletzt muss darauf hingewiesen werden, dass hier als Maß für den beruflichen Erfolg lediglich das erzielte Einkommen berücksichtigt wurde. Ob die gefundenen Zusammenhänge auch für weitere Dimensionen wie etwa das Arbeitslosigkeitsrisiko, innerbetriebliche Beförderungschancen, das sozioökonomische Prestige oder die Arbeitszufriedenheit gelten, bleibt offen. Weitere interessante Aspekte, die in diesem Beitrag hauptsächlich aufgrund der beschränkten Datenlage außer Acht gelassen wurden, sind u.a. die Fragen, ob der gefundene Offizierseffekt einer zeitlichen Entwicklung unterworfen ist, ob er sich nach den regionalen, durch die Sprache geprägten kulturellen Rahmenbedingungen innerhalb der Schweiz unterscheidet und wie sich der militärische Rang auf den Erfolg von beruflich Selbständigen auswirkt.

\section{Anhang}

Kennzahlen der Variablen in den Regressionsmodellen (Standardabweichung in Klammern)

\begin{tabular}{lcccc}
\hline Variable & $\begin{array}{c}\text { arithmetisches Mittel } \\
\text { Modelle 1-8 } \\
(N=579)\end{array}$ & $\begin{array}{c}\text { Modelle 9-10 } \\
(N=702)\end{array}$ & Min. & Max. \\
\hline Netto-Stundenlohn (logarithmiert) & 3.509 & 3.493 & 1.1 & 4.8
\end{tabular}

Militärischer Rang (RG: Soldat):

- kein Militärdienst

$\begin{array}{rrrr} & 0.175 & 0.0 & 1.0 \\ 0.193 & 0.160 & 0.0 & 1.0 \\ 0.074 & 0.061 & 0.0 & 1.0 \\ 11.977 & 11.921 & 8.0 & 17.5\end{array}$

- Unteroffizier

(2.375)

- Offizier

Berufserfahrung

Arbeitsanstrengung

0.401

0.415

0.0

Führungsposition

0.699

0.708

0.0

Stellenfindung über soziale Kontakte

0.245

0.258

0.0

Stellenfindung (RG: formell):

- starke soziale Kontakte

- schwache soziale Kontakte

0.100

0.0 
Kennzahlen der Variablen in den Regressionsmodellen (Standardabweichung in Klammern)

\begin{tabular}{|c|c|c|c|c|}
\hline \multirow[b]{2}{*}{ Variable } & \multicolumn{2}{|c|}{ arithmetisches Mittel } & \multirow[t]{2}{*}{ Min. } & \multirow[t]{2}{*}{ Max. } \\
\hline & $\begin{array}{c}\text { Modelle } 1-8 \\
\quad(N=579)\end{array}$ & $\begin{array}{l}\text { Modelle } 9-10 \\
\quad(N=702)\end{array}$ & & \\
\hline \multicolumn{5}{|l|}{ Branchen (RG: Industrie): } \\
\hline - Land-/Forstwirtschaft & & 0.027 & 0.0 & 1.0 \\
\hline - Baugewerbe & & 0.115 & 0.0 & 1.0 \\
\hline - Gastgewerbe/Handel/Reparatur & & 0.087 & 0.0 & 1.0 \\
\hline - Verkehr/Nachrichten & & 0.050 & 0.0 & 1.0 \\
\hline - Banken/Finanzgesellschaften & & 0.051 & 0.0 & 1.0 \\
\hline - Versicherung/Beratung & & 0.061 & 0.0 & 1.0 \\
\hline - sonstige Dienstleistungen & & 0.248 & 0.0 & 1.0 \\
\hline - öffentliche Verwaltung & & 0.105 & 0.0 & 1.0 \\
\hline
\end{tabular}

Anmerkungen: Abhängig erwerbstätige Männer mit Schweizer Staatsbürgerschaft im Alter von 18-70 Jahren, ohne Lehrlinge und noch nicht Ausgehobene; Modelle 1-8: nur Personen, die Militärdienst leisten bzw. geleistet haben; RG: Referenzgruppe; Quelle: SAMS 98, ungewichtet.

\section{Literatur}

Ambühl, I., 1998: Muntere Seilschaft. Armeefilz in Basel: Auffällig viele höhere Staatsdiener verfügen über ein Offizierspatent. SonntagsZeitung vom 31. Mai: 8.

Becker, G.S., 1993 [1964]: Human Capital: A Theoretical and Empirical Analysis with Special Reference to Education, $3^{\text {rd }}$ ed. Chicago: Univ. of Chicago Press.

Belsley, D.A. / Kuh, E. / Welsch, R.E., 1980: Regression Diagnostics: Identifying Influential Data and Sources of Collinearity. New York: John Wiley \& Sons.

Berner, S., 1994: Einstellungs- und Beförderungsaussichten von Milizoffizieren in multinationalen Unternehmen in der Schweiz - heutiger Stand und absehbare Veränderungen im Zusammenhang mit der „Armee 95“. Diplomarbeit an der Hochschule St. Gallen (erhältlich bei der Eidgenössischen Militärbibliothek).

Bertossa, L. / Binzegger, D. / Bühler, S., 1994: Das Gewicht der militärischen Ausbildung und Karriere. Schweizerische Arbeitgeber-Zeitung 89 (3): 65-69.

Bollen, K.A. / Jackman, R.W., 1990: Regression Diagnostics: An Expository Treatment of Outliers and Influential Cases. S. 257-291 in: J. Fox / J.S. Long (ed.): Modern Methods of Data Analysis. Newbury Park: Sage Publ.

Bonjour, D., 1997: Lohndiskriminierung in der Schweiz. Eine ökonometrische Untersuchung. Bern: Haupt.

Bonjour, D. / Gerfin, M., 1995: Einkommensungleichheit zwischen Frauen und Männern. Eine ökonometrische Analyse der Schweizer Arbeitskräfteerhebung: Kommentar. Schweizerische Zeitschrift für Volkswirtschaft und Statistik 131: 701-710.

Bourdieu, P., 1983: Ökonomisches Kapital, kulturelles Kapital, soziales Kapital. Soziale Welt 34: 183-198.

Bridges, W.P. / Villemez, W.J., 1986: Informal Hiring and Income in the Labor Market. American Sociological Review 51: 574-582.
Brüderl, J. / Diekmann, A. / Engelhardt, H., 1993: Einkommensunterschiede zwischen Frauen und Männern in der Schweiz. Eine Zerlegung des Einkommensabstandes mit der Komponentenmethode. Schweizerische Zeitschrift für Soziologie 19: 573-588.

Brunner, D., 1997: Sind militärische Führungsgrundsätze in der Wirtschaft noch anwendbar? Allgemeine Schweizerische Militärzeitschrift 163 (11): 12.

Bundesamt für Statistik, 1999a: Bildungsabschlüsse 1997. Neuchâtel.

Bundesamt für Statistik, 1999b: Schülerinnen, Schüler und Studierende 1997/98. Neuchâtel.

Coleman, J.S., 1988: Social Capital in the Creation of Human Capital. American Journal of Sociology 94 (Supplement): S95-S120.

De Graaf, N.D. / Flap, H.D., 1988: „With a Little Help from My Friends": Social Resources as an Explanation of Occupational Status and Income in West Germany, The Netherlands, and the United States. Social Forces 67: 452-472.

Diekmann, A. / Engelhardt, H., 1995: Einkommensungleichheit zwischen Frauen und Männern. Eine ökonometrische Analyse der Schweizer Arbeitskräfteerhebung. Schweizerische Zeitschrift für Volkswirtschaft und Statistik 131: 57-83.

Diekmann, A. / Engelhardt, H. / Jann, B. / Armingeon, K. / Geissbühler, S., 1999: Der Schweizer Arbeitsmarktsurvey 1998. Codebuch und Dokumentation. Universität Bern.

DuMouchel, W.H. / Duncan, G.J., 1983: Using Sample Survey Weights in Multiple Regression Analyses of Stratified Samples. Journal of the American Statistical Association 78: 535-543.

Erb, W., 1997: Will die Wirtschaft keine Offiziere mehr? AOG-Mitteilungen (Allgemeine Offiziersgesellschaft von Zürich und Umgebung) 65 (1): 12-14.

Falter, J.-M. / Ferro Luzzi, G., 2000: Public - Private Sector Wage Differentials in Switzerland. Schweizeri- 
sche Zeitschrift für Volkswirtschaft und Statistik 136: 319-339.

Feld, S. L., 1997: Network analysis using the method of path lists: testing the weak ties hypothesis. Social Networks 19: 97-111.

Flap, H. / Völker, B., 2001: Goal specific social capital and job satisfaction. Effects of different types of networks on instrumental and social aspects of work. Social Networks 23: 297-320.

Fopp, A., 1992: Die Bedeutung der schweizerischen Generalstabsausbildung für die Problemlösungsfähigkeit ziviler Führungskräfte. Diplomarbeit an der Hochschule St. Gallen (erhältlich bei der Eidgenössischen Militärbibliothek).

Franz, W. 1996 [1991]: Arbeitsmarktökonomik, 3. Aufl. Berlin: Springer.

Franzen, A., 2001: Wages and the Use of New Technologies: An Empirical Analysis of the Swiss Labor Market. Schweizerische Zeitschrift für Volkswirtschaft und Statistik 137: 505-523.

Ganzeboom, H.B.G. / Treiman, D. J., 1996: Internationally Comparable Measures of Occupational Status for the 1988 International Standard Classification of Occupations. Social Science Research 25: 201-239.

Granovetter, M.S., 1973: The Strength of Weak Ties. American Journal of Sociology 78: 1360-1380.

Granovetter, M.S., 1974: Getting a Job. A Study of Contacts and Careers. Cambridge, Mass.: Harvard Univ. Press.

Granovetter, M.S., 1983: The Strength of Weak Ties: A Network Theory Revisited. Sociological Theory 1: 201-233.

Hallerbach, R., 1988: Erfahrungen eines Großunternehmens. Aufstieg im Zivilberuf für Zeitoffiziere der Bundeswehr. Europäische Wehrkunde 37 (6): 330-331.

Haltiner, K.W. / Wenger, A. / Bennet, J. / Szvircsev, T., 2001: Sicherheit 2001. Außen-, Sicherheits- und Verteidigungspolitische Meinungsbildung im Trend. Zürich: ETHZ.

Hartmann, M., 1996: Topmanager. Die Rekrutierung einer Elite. Frankfurt a. M./New York: Campus.

Henneberger, F. / Sousa-Poza, A., 1999: Geschlechtsspezifische Lohndiskriminierung: Neueste Evidenz von den Mikrodaten aus der Schweizerischen Arbeitskräfteerhebung. Schweizerische Zeitschrift für Soziologie 25: 259-279.

Hochstrasser, J.-M., 1989: Die berufliche Situation der Offiziere der Felddivision 6. Historisches Seminar der Universität Zürich (erhältlich bei der Eidgenössischen Militärbibliothek).

Hollenstein, H., 1987: Spitzenmanager in der Schweiz. Herkunft, Ausbildung, Werdegang, Stellung in der Gesellschaft. Bern: Haupt.

Ibarra, H., 1992: Homophily and Differential Returns: Sex Differences in Network Structure and Access in an Advertising Firm. Administrative Science Quarterly 37: $422-447$.

Jann, B., 2003: The Swiss Labor Market Survey 1998 (SLMS 98). Erscheint in: Journal of Applied Social Science Studies 123.
Keller, D., 1995: Manager ohne Offiziershut. Diplomarbeit an der ETH Zürich (erhältlich bei der Eidgenössischen Militärbibliothek).

Keller, R. / Wigger, B., 1998: Umfrage in Armee und Wirtschaft: Die höhere Kaderausbildung im Wandel. Forum - Magazin für das Instruktionskorps Nr. 30: 19-21.

Killingsworth, M.R., 1983: Labor Supply. Cambridge, Mass.: Cambridge Univ. Press.

Kriesi, H., 1980: Entscheidungsstrukturen und Entscheidungsprozesse in der Schweizer Politik. Frankfurt am Main: Campus.

Kugler, P., 1988: Lohndiskriminierung in der Schweiz: Evidenz von Mikrodaten. Schweizerische Zeitschrift für Volkswirtschaft und Statistik 124: 23-47.

Lichtsteiner, R., 1997: Vom Nutzen der militärischen Kaderausbildung für ein international tätiges Industrieunternehmen. AOG-Mitteilungen (Allgemeine Offiziersgesellschaft von Zürich und Umgebung) 65 (1): 9-11.

Lin, N. / Ensel, W.M. / Vaughn, J.C., 1981a: Social Resources and the Strength of Ties: Structural Factors in Occupational Status Attainment. American Sociological Review 46: 393-405.

Lin, N. / Vaughn, J.C. / Ensel, W.M., 1981b: Social Resources and Occupational Status Attainment. Social Forces 59: 1163-1181.

Liu, W.T. / Duff, R.W., 1972: The Strength in Weak Ties. Public Opinion Quarterly 36: 361-366.

Lutz, N., 1998: Ende einer amour fou. GSoA-Zitig Nr. 74: 6. Marsden, P.V. / Campbell, K.E., 1984: Measuring Tie Strength. Social Forces 63: 482-501.

Marsden, P.V. / Hurlbert, J.S., 1988: Social Resources and Mobility Outcomes: A Replication and Extension. Social Forces 66: 1038-1059.

Mincer, J., 1974: Schooling, Experience, and Earnings. New York: Columbia Univ. Press.

Montgomery, J.D., 1991: Social Networks and LaborMarket Outcomes: Toward an Economic Analysis. The American Economic Review 81: 1408-1418.

Montgomery, J.D., 1992: Job Search and Network Composition: Implications of the Strength-Of-Weak-Ties Hypothesis. American Sociological Review 57: 586596.

Moore, G. / White, D., 2001: Geschlechterungleichheit und Elite-Netzwerke in vierundzwanzig Industriegesellschaften. S. 136-151 in: B. Heintz (Hrsg.): Geschlechtersoziologie. Sonderheft 41 der Kölner Zeitschrift für Soziologie und Sozialpsychologie. Wiesbaden: Westdeutscher Verlag.

Mueller, U. / Mazur, A., 1996: Facial Dominance of West Point Cadets as a Predictor of Later Military Rank. Social Forces 74: 823-850.

Murray, S.O. / Rankin, J.H. / Magill, D.W., 1981: Strong Ties and Job Information. Sociology of Work and Occupations 8: 119-136.

Oester, E.B., 1996: Listen der Macht: Vom Männerbund zum Old Boy's Network. Schriftenreihe Nr. 0996.1, Institut für Soziologie der Universität Bern.

Podolny, J.M. / Baron, J.N., 1997: Resources and Relationships: Social Networks and Mobility in the Workplace. American Sociological Review 62: 673-693. 
Preisendörfer, P. / Voss, T., 1988: Arbeitsmarkt und soziale Netzwerke. Die Bedeutung sozialer Kontakte beim Zugang zu Arbeitsplätzen. Soziale Welt 39: 104-119.

Schmitt, P.A., 1992: Militär als Karrierehilfe: Helm ab zum Aufstieg. Bilanz? (7): 89-92.

Spälti, P., 1990: Führen in Armee und Wirtschaft. Allgemeine Schweizerische Militärzeitschrift 156 (11): 679-684.

Spälti, P., 1995: Militärische Weiterausbildung vs. zivile Karriere? Allgemeine Schweizerische Militärzeitschrift 161 (7/8): 23-24.

Staffelbach, B., 1994: Zivile und militärische Karriere? Allgemeine Schweizerische Militärzeitschrift 160 (10): 9-12.

Staffelbach, B., 1998: Nachfragekonkurrenz zwischen Wirtschaft und Armee. Neue Zürcher Zeitung 219 (135): 11.

Vontobel, H.-D., 1992: Der Nutzen militärischer Führungstätigkeit für den zivilen Beruf. Neue Zürcher Zeitung 213 (244): 23.

Wegener, B., 1987: Vom Nutzen entfernter Bekannter. Kölner Zeitschrift für Soziologie und Sozialpsychologie 39: 278-301.

Wegener, B., 1989: Soziale Beziehungen im Karrierepro- zess. Kölner Zeitschrift für Soziologie und Sozialpsychologie 41: 270-297.

Wegener, B., 1991: Job Mobility and Social Ties: Social Resources, Prior Job, and Status Attainment. American Sociological Review 56: 60-71.

White, H., 1980: A Heteroskedasticity-Consistent Covariance Matrix Estimator and a Direct Test for Heteroskedasticity. Econometrica 48: 817-838.

Willis, R.J., 1992 [1986]: Wage Determinants: A Survey and Reinterpretation of Human Capital Earnings Functions. S. 525-602 in: O. Ashenfelter / R. Layard (ed.): Handbook of Labor Economics, Vol. 1, $2^{\text {nd }}$ ed. Amsterdam: North-Holland.

Winship, C. / Morgan, S.L., 1999: The Estimation of Causal Effects from Observational Data. Annual Review of Sociology 25: 659-707.

Winship, C. / Radbill, L., 1994: Sampling Weights and Regression Analysis. Sociological Methods and Research 23: 230-257.

Zimmermann, G. / Meyer, U. / Rub, G. / Capaul, M., 1992: Miliär \& Karriere. Höhere Wirtschafts- und Verwaltungsschule (erhältlich bei der Eidgenössischen Militärbibliothek).

Summary: In Switzerland it has often been argued, at least until recently, that a career in the armed forces is greatly beneficial to professional success in civilian life. Despite the fact that, as a peculiarity of the Swiss militia system, professional and military careers are interwoven in the biographical process, the actual influence of a military career on professional success has yet to be empirically verified. Moreover, it is also necessary to ask what the possible reasons for this might be. Consequently, the first goal of this paper is to examine empirically whether a military career has a positive effect on a civilian career. Second, possible explanations will be discussed. In particular, self selection, the acquisition of human capital (leadership qualities), and the establishment of an effective social network will be considered. The analyses which are based on the Swiss Labor Market Survey 1998 show, first of all, that higher military ranks go hand in hand with higher monetary income even when controlling for education and work experience. Second, the income premium does not seem to be based on mechanisms of self-selection nor on the acquisition of particularly profitable human capital. Rather it is to be assumed that the greater professional success of Swiss militia officers is due to their rich social network of "valuable" ties. 